\title{
Studies on the key methods for compressive ghost-image tracking based on background subtraction
}

\author{
${ }^{1}$ Zhang Leihong, ${ }^{1} *$ Kang Yi, ${ }^{1}$ Li Bei, ${ }^{1}$ Zhan Wenjie, ${ }^{1}$ Zhang Dawei and \\ ${ }^{2}$ Ma Xiuhua \\ ${ }^{1}$ University of Shanghai for Science and Technology, Shanghai, China, 200093 \\ ${ }^{2}$ Shanghai Institute of Optics and Fine Mechanics, CAS, Shanghai, China, 201800 \\ *ky930827@sina.com
}

Received: 13.02 .2017

\begin{abstract}
Efficient object tracking represents a technology important for many vision applications. It is known that ghost imaging (GI) has a great potential if compared with a standard imaging and solves many problems in case if the common object tracking cannot be carried out. Here we show how the techniques of compressive GI and background subtraction can achieve object tracking. First, object information is captured with the GI. A characteristic measured for an object is obtained by subtracting background in the compressed domain. This characteristic uses compressive sensing to reconstruct the object image. Then the object image is projection-positioned to obtain the corresponding centroid coordinates. At last, the object trajectory is recovered with a polynomial fit, thus providing successful object tracking. Our simulation experiments suggest that the technique can track objects accurately under condition of low sampling ratios. Moreover, it decreases drastically the number of measurements needed for reconstruction and improves the tracking efficiency.
\end{abstract}

Key words: object tracking, compressive sensing, ghost imaging, background subtraction

PACS: $42.30 . \mathrm{Wb}$

UDC: 004.932 .1

\section{Introduction}

Object tracking technologies are very important for the field of computer vision. It has been widely used in vehicle tracking, video surveillance, human-computer interaction and so on [1]. Although a lot of efficient object-tracking algorithms have been proposed, many problems in their application still need to be solved. For instance, it is matter of importance whether a whole object or its part is covered. Illumination changes in the environment, changes occurring with objects and a background noise can be mentioned among the other problems. As a result, it is still difficult to design the object-tracking algorithm which is accurate, reliable and stabile for a long time [2].

To track a moving object, we first need to detect it. In case of target-detection techniques, the common methods are a background subtraction (BS), an optical-flow method, and a frame difference one $[3,4]$. If compared with the other methods, the BS is simple to implement in the context of fixed or slowly changing background. Then moving objects can be detected quickly and accurately, which is suitable for video surveillance [5].

Area matching, feature matching, template matching and contour matching are among the common methods used in the process of tracking [6, 7]. In view of the problems arising in this process, the authors of the work [8] have put forward an object tracking based on multi-feature 
fusion. It is able to adapt to illumination changes though involves complex calculations. The study [9] has suggested a technique for object tracking which is based on a scale-invariant feature. To some extent, it can overcome the problems of similar appearance of the object near the target and achieves the main goal of stable tracking. Nonetheless, the above technique is easily affected by the target scale and velocity changes.

Traditional techniques used for object tracking have also shortcomings associated with data redundancy and bad tracking performance in harsh environments. In this respect, a compressive sensing (CS) [10] can use the sampling frequency far lower than the Nyquist sampling restrictions imposed upon signal restoration. In particular, application of the CS in the spectral-reflectance reconstruction improves the reconstruction accuracy so that the image can be restored more accurately $[11,12]$. To improve the reconstruction accuracy and reduce workload, an adaptive CS has been used in the work [13] to reconstruct the spectral reflectance. In Ref. [14], the CS has been applied to target tracking. In this way, fast feature extraction and real-time object tracking have been achieved. Although the technique reduces the problem of target loss caused by a drift in the process of tracking, the problems with environment and illumination changes still have a great influence on the tracking. It is difficult to achieve the tracking effect stable for long enough times. Moreover, another problem linked with bad tracking performance in the harsh environment has not been solved, too.

The authors of Ref. [15] have presented a technique that allows for direct reconstruction of background-subtracted images, using a CS theory and a complementary-modulation technique. Though it is really robust against changing illumination and noises, this technique still reveals flaws in some special environments. On the other hand, a ghost image (GI) technique [16] enables obtaining object images, using the optical paths external with respect to the objects themselves. A method combining entangled-photon approach with the CS has been proposed in Ref. [17] to detect different targets. Here the number of samplings and the redundancy in the transmission process are reduced and, moreover, the tracking performance remains sufficiently good in the harsh environment. Nonetheless, the technique is difficult to implement because of complicated equipment required. Finally, the authors of the work [18] have suggested using entangled photons and compression sensing. The technique demonstrates robustness with respect to the environment but remains difficult to implement in practice. Basing on the said above, we believe that a combination of the BS and compressive GI methods, which we refer to as CSGI, can reduce the data redundancy and manifest a sufficient tracking performance in the harsh environment. This can improve significantly the tracking.

As a consequence, the present work presents a technique aimed at object tracking, which is based on both the compressive GI and the BS. It is termed hereafter as a BSCSGI technique. According to this technique, we first collect the image information with the GI. Then the object information is obtained using the BS in the compressed domain. Finally, the object is reconstructed directly using the CS. Since the reconstructed image contains only the moving object at each time, the very problem of object tracking becomes essentially simpler. The location information referred to different time moments can be deduced from the reconstructed images. Then the object trajectory can be calculated issuing from this information, and the next positions of the object can be predicted. The location area predicted can be reconstructed directly with the CSGI and, therefore, a small-area image containing the object can be obtained. Our technique can use the sampling frequencies which are far lower than those associated with the Nyquist sampling restrictions, thus enabling one to restore the target object more accurately. The BSCSGI can solve 
many problems which are otherwise difficult to overcome with the conventional imaging techniques. As an example, the number of samplings can be reduced drastically when our technique is employed in the object tracking. Besides of simplicity of the BSCSGI, it improves the accuracy of object tracking and reduces the influence of noises.

\section{Theory of compressive GI}

In the computational GI, light irradiates a spatial light modulator which modulates the intensity of the emergent light. Then the modulated light field falls upon the object under test. The bucket detector collects all the light having passed through the object, and some resulting bucket-detector reading is obtained. After $N$ samplings, $N$ detector readings $D_{i}$ are collected. A speckle field $I_{i}(x, y)$ generated by the spatial light modulator is recorded for each modulation act. Then the second-order correlation function is got due to correlating operation applied to the speckle fieldintensity distribution $I$ and the bucket-detector readings $D$. The image of the target object $G(x, y)$ is given by the formula

$$
G(x, y)=\left\langle I_{i}(x, y) D_{i}\right\rangle-\left\langle I_{i}(x, y)\right\rangle\left\langle D_{i}\right\rangle \quad i=1,2 \cdots N,
$$

where \langle\rangle implies averaging.

The CS theory points out that the signal length $N$ in a known transform domain $\psi$ is sparse. Then one can obtain the coefficients of the signal measured linearly in the transform domain, using a $m \times n(m<<n)$-dimension measurement matrix $\phi$, which is not related to the transformation basis $\psi$. As a result, we have the measured values $Y$ with the dimension $m \times 1$. Hence, the dimension of the measured values is less than that of the unknown signal.

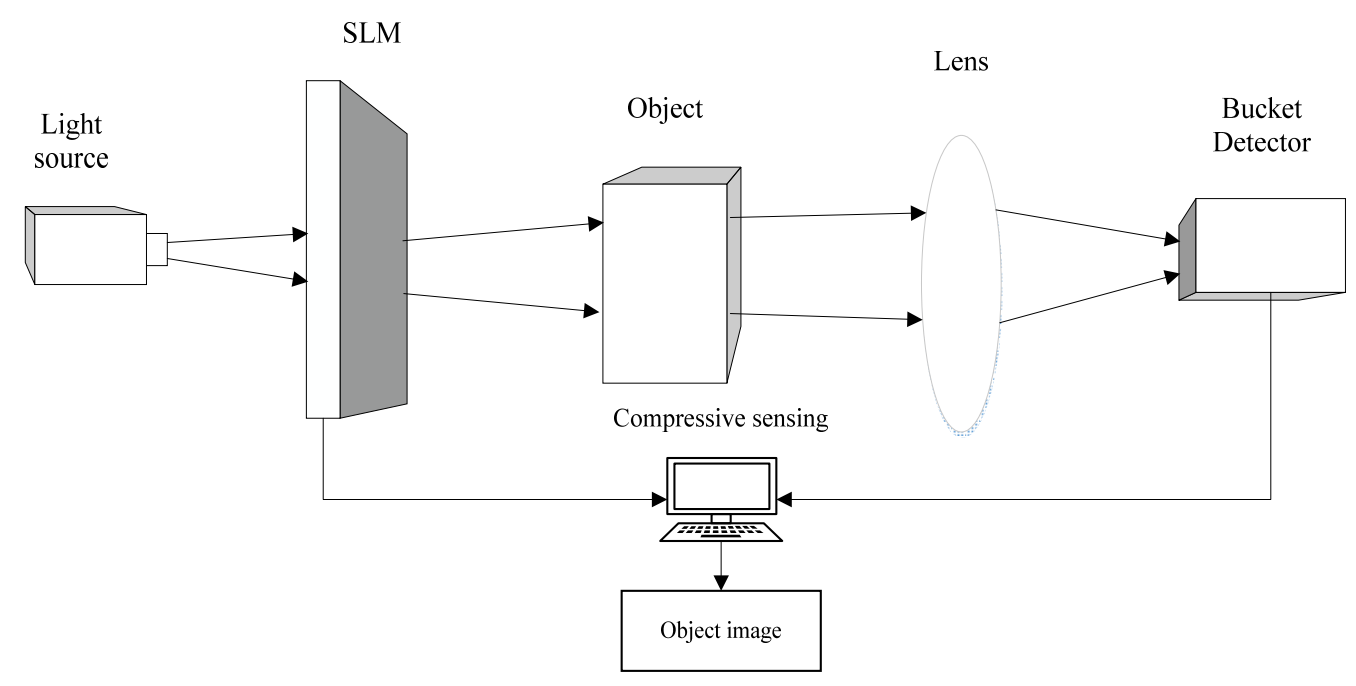

Fig. 1. Schematic diagram of compressive GI.

In the traditional theory of signal recovery, a signal cannot be found exactly. According to the CS theory, we make use of the measured values $Y$, the transform base $\psi$ and the measurement matrix $\phi$. Then the original signal can be reconstructed with arbitrarily high precision by solving the optimization problem. The CS theory can be applied to the GI systems, as 
shown in Fig. 1. The bucket detector yields $N$ readings for the CS and $N$ optical-intensity distributions $I(x, y)$ as a measurement matrix. Then the compressive GI model can be constructed as

$$
T_{c s}=T ; \min \|\psi\{T(x, y)\}\|_{L_{l}},
$$

subject to

$$
\mathrm{D}_{\mathrm{i}}=\int \operatorname{dxdyI}_{\mathrm{i}}(\mathrm{x}, \mathrm{y}) \mathrm{T}(\mathrm{x}, \mathrm{y}) ; \forall_{\mathrm{i}}=1, \ldots, \mathrm{N},
$$

where $T_{c s}$ represents the image of the target object, $T(x, y)$ the projection function of the object, $\|\cdot\|_{L_{1}}$ the $L_{1}$ norm, and $\psi\{T(x, y)\}$ the sparse matrix of the object.

\section{Principles of compressive GI-based object tracking that involves BS}

The compressive GI-based object tracking that involves the BS include the two principles. The first is object detection based on the compressive GI and the BS. A reconstructed image which contains only the object is thus obtained. The second moment is the object tracking itself. Here the object reconstructed with the CS undergoes projection positioning to obtain a centroid coordinate. Then an object trajectory is built with a polynomial fit. Finally, the position of the object at the next moment can be predicted, thus narrowing the predicted area, which is reconstructed using the compressive GI. In this manner, the object can be successfully tracked and a small-area image containing the object can be obtained.

According to the principles of CSGI, a laser light is modulated by the spatial light modulator in the process of target detection. The modulated light passes through the background image $X_{b}$ and then through the test image $X_{t}$. The modulated light is collected by a lens and received by the bucket detector. After $M$ samplings, $M$ values of the background image $D_{t}(i=1,2 . . M)$ and $M$ values of the test image $D_{i}(i=1,2 \ldots M)$ are detected. Each light-intensity distribution is stored and, as a result, a set of transformed stored results represents a so-called measurement matrix of the CS.

To extract the moving object, one has to remove any background (i.e., static) elements. Both of the $X_{b}$ and $X_{t}$ quantities contain the same elements, the difference being that $X_{t}$ contains only the target object and $X_{b}$ only the background elements. Therefore the target-object image detected with the BS represents a difference between $X_{b}$ and $X_{t}$. Then the information acquired from the object is sparse, thus conforming to the main operation principles of the CS. Following from the $X_{b}$ and $X_{t}$ values measured with the GI, we obtain the $D_{t}$ and $D_{t}$ parameters associated respectively with $X_{b}$ and $X_{t}$. The resulting BS-based parameters of the target object are given by

$$
D_{d}=D_{t}-D_{b} .
$$

Here $D_{t}$ denote the values measured after subtracting, which are nothing but the values measured for the target object. Then the image can be reconstructed with the CSGI procedure. Fig. 2a and Fig. 2b correspond to the test and background images. As shown in Fig. 2c, the object obtained using the background-difference method is sparse, so that the numbers of necessary measurements and reconstructions are less than those of the background and test images. In this way the reconstruction efficiency is improved and the tracking becomes more reliable.

Finally, a standard computer image-processing technology is used to carry out binarization of a reconstructed object image under a suitable threshold for image segmentation (see Fig. 2d). Then the binary object image suffers a projection positioning to obtain a smallest outer rectangle of the 


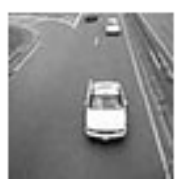

a)

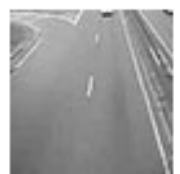

b)

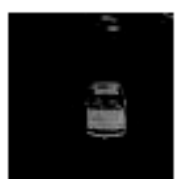

c)

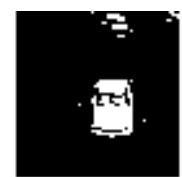

d)

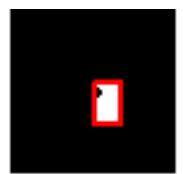

e)

Fig. 2. Test image (a), background image (b), object image reconstructed with CSGI (c), (d) binary image, and (e) object image obtained after projection positioning.

object, with a rectangle centre being a centroid of the object (see Fig. 2e). The projection positioning uses projections along the two directions of image, horizontal and vertical, so as to find out the positions and the ranges of the image along these two directions. The specific method used by us is a top-down progressive scan of the image. The relevant sum of the columns is as follows:

$$
f\left(y_{i}\right)=\sum_{i}^{N} f\left(x_{i}, y_{i}\right) .
$$

After analyzing the horizontal-projection results, one finds that larger $f\left(y_{i}\right)$ 's correspond to the object area, while zero or smaller $f\left(y_{i}\right)$ 's to the background area or the noise. Having this feature in mind, one can determine the upper and lower boundaries. The left and right boundaries of the object can be determined in the same manner. The smallest outer rectangle of the object is determined by the four boundary lines mentioned above. Then the central position of the upper and lower boundaries is the ordinate of the object centroid, whereas the central position of the left and right boundaries is the abscissa of this centroid. The object trajectory $Y=f(X, C)$ can be calculated with a standard polynomial fit. Here $X$ and $Y$ are the column vectors composed respectively of abscissas and ordinates of the object centroids, and $C$ is some indeterminate multinomial coefficient. A continuous curve can be found after analyzing the trajectory, which can, in principle, be arbitrarily close to the discrete points of the true object trajectory. Here we take the analytic expression of the polynomial as a model and employ the least-squares method [19] to measure the approximation effect, as described below.

The moving trajectory of the object should be found as

$$
Y=f(X, C)=c_{0}+c_{1} X+c_{2} X^{2}+\ldots c_{n} X^{n},
$$

where $C=\left\{c_{0}, c_{1}, c_{2} \ldots c_{(n-1)}\right\}$ is the vector of $n$ parameters of the polynomial. Then we calculate the variance of the $N$ known points and the estimated fitting curve:

$$
\|\delta\|_{2}^{2}=\sum_{i=1}^{N}\left[y_{i}-\left(c_{0}+c_{i} x_{i}+c_{2} x_{i}^{2}+\ldots c_{n} x_{i}^{n}\right)\right]^{2} .
$$

Taking partial derivative of each $C_{i}$ in the variance $\|\delta\|_{2}^{2}$, we arrive at the matrix

$$
\left[\begin{array}{cccc}
N & \sum_{i=1}^{N} x_{i} & \cdots & \sum_{i=1}^{N} x_{i}^{n} \\
\sum_{i=1}^{N} x_{i} & \sum_{i=1}^{N} x_{i}^{2} & \cdots & \sum_{i=1}^{N} x_{i}^{n+1} \\
\vdots & \vdots & \ddots & \vdots \\
\sum_{i=1}^{N} x_{i}^{n} & \sum_{i=1}^{N} x_{i}^{n+1} & \cdots & \sum_{i=1}^{N} x_{i}^{2 n}
\end{array}\right]\left[\begin{array}{l}
c_{0} \\
c_{1} \\
\vdots \\
c_{n}
\end{array}\right]=\left[\begin{array}{c}
\sum_{i=1}^{N} y_{i} \\
\sum_{i=1}^{N} x_{i} y_{i} \\
\vdots \\
\sum_{i=1}^{N} x_{i}^{n} y_{i}
\end{array}\right] .
$$

Basing on this matrix, all of the parameters $C=\left\{c_{0}, c_{1}, c_{2} \ldots c_{(n-1)}\right\}$ of the least-squares fitted trajectory can be obtained. 


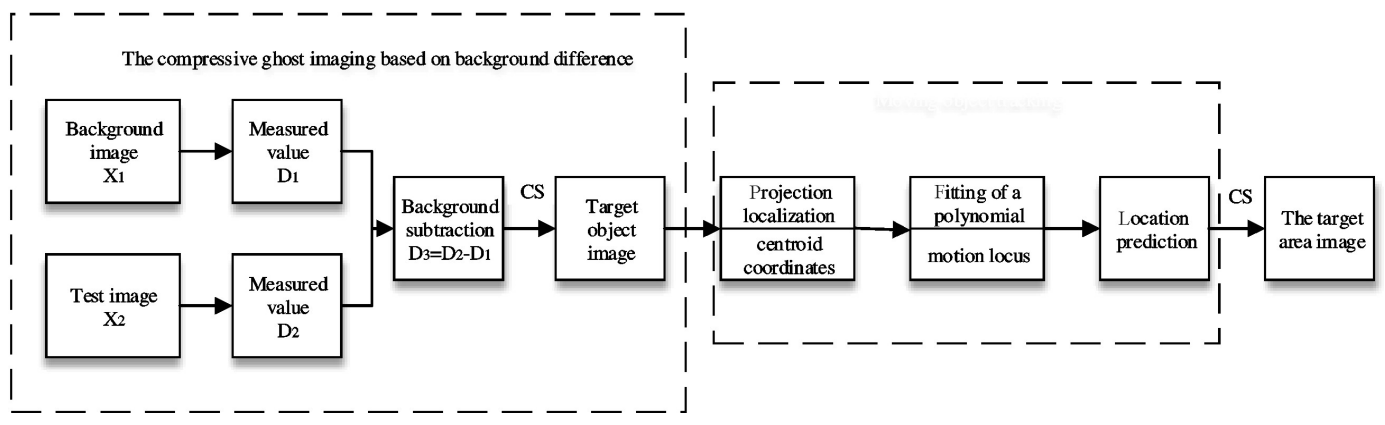

Fig. 3. Flow diagram of BSCSGI.

Having known the object trajectory, one can predict the centroid coordinate of the object $\left(x^{\prime}, y^{\prime}\right)$ and calculate the smallest outer rectangle of the object. Notice that the compressive GI is used to reconstruct the image in the smallest outer rectangle so that the image containing only the moving object can be obtained. Since the predicted results shrink the scope of imaging at every moment, the image-measurement and image-reconstruction times are greatly reduced, along with complexity of the object tracking. Fig. 3 illustrates a flow diagram of the BSCSGI technique.

\section{Simulation results}

We verified the performance of the BSCSGI technique through numerical simulations. In our simulation experiments, a random matrix was used as a measurement matrix. Grayscale images were adopted from traffic.avi, a video provided with MATLAB. The first frame was taken as a background image and the other continuous frames were selected as test images. To facilitate the simulation experiments, we set the size of both binary and grayscale images to be $64 \times 64$ pixels. In our simulations, modulated laser light fell upon the object to generate a bucket-detector reading, which was recorded as a single-time sampling. The sampling ratio was equal to the ratio of number of samplings and the total number of pixels in the image. The simulation platform was MATLAB software. The images were reconstructed using a known algorithm of orthogonal matching pursuit. The mean-square error was selected as a measuring standard in order to analyze quantitatively the image-reconstruction effect.

\subsection{Confirmation of feasibility of the technique}

Here we imply to verify the feasibility of the BSCSGI. First the object-detection procedure, which represents the initial step in the object tracking, needs to be verified. In Fig. 4, the sampling rate of the reconstructed image is $50 \%$. We find that the object image can be reconstructed with the BSCSGI technique under low-sampling conditions. Therefore our method can detect the objects accurately enough.

Now the feasibility of the object detection with the BSCSGI should be verified. After the BSCSGI is used to track some object, its trajectory can be built. The image reconstructed using the BSCSGI is transformed into a binary image with the aid of a standard technology. Then the binary images obtained are subjected to projection positioning to obtain the smallest outer rectangle of the object. As a result, a relevant centroid and a length and width of the object are recorded. Fig. 5 displays the results obtained when the projection positioning is applied to the binary image. Table 1 summarizes the object-centroid coordinates. As seen from Table 1, the maximum errors for the length and the width of projection positioning are about 1 pixel, and the same is true of the maximum error of the reconstructed centroid coordinates. Hence, the results derived with the 


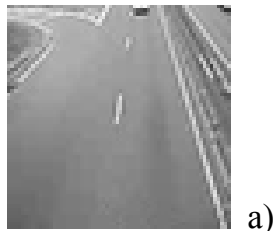

a)

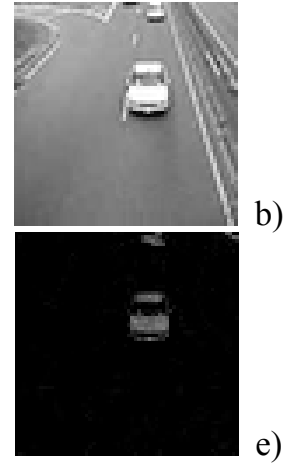

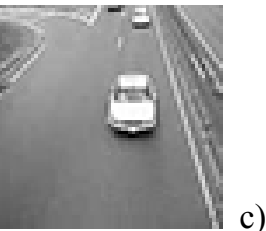

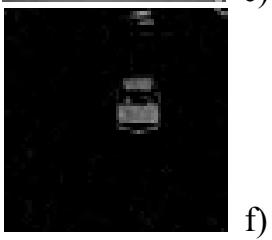

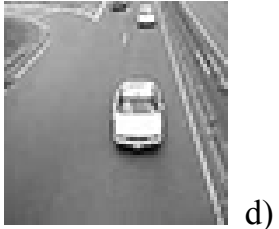

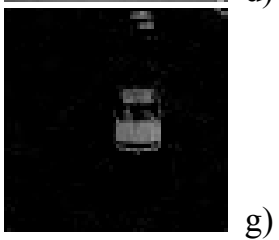

)

g)

Fig. 4. Background image (a), three continuous frames (b)-(d), and images reconstructed with CSGI (e)-(g)

projection positioning agree well with the corresponding actual values, such that our projectionpositioning procedures are indeed reliable. After the centroid coordinate are known, one can built the object trajectory using the polynomial fit.

Table 1. Actual coordinates of a moving object and coordinates reconstructed with our technique. The coordinates, the length and the width are measured in the units of pixels, where a lower left corner of the image is taken as an origin.

\begin{tabular}{c|c|c|c|c}
\hline & & Frame \#1 & Frame \#2 & Frame \#3 \\
\hline \multirow{2}{*}{ Actual values } & $\begin{array}{c}\text { Centroid } \\
\text { coordinates }\end{array}$ & $(24.0,38.5)$ & $(28.0,39.0)$ & $(31.0,38.0)$ \\
\cline { 2 - 5 } & $\begin{array}{c}\text { Length and } \\
\text { width }\end{array}$ & $14.0,11.9$ & $16.0,12.0$ & $18.0,12.0$ \\
\hline \multirow{2}{*}{$\begin{array}{c}\text { Reconstructed } \\
\text { values }\end{array}$} & $\begin{array}{c}\text { Centroid } \\
\text { coordinates }\end{array}$ & $(23.5,38.5)$ & $(27.0,38.5)$ & $(30.0,37.5)$ \\
\cline { 2 - 5 } & $\begin{array}{c}\text { Length and } \\
\text { width }\end{array}$ & $15.0,11.0$ & $16.0,13.0$ & $18.0,11.0$ \\
\hline
\end{tabular}
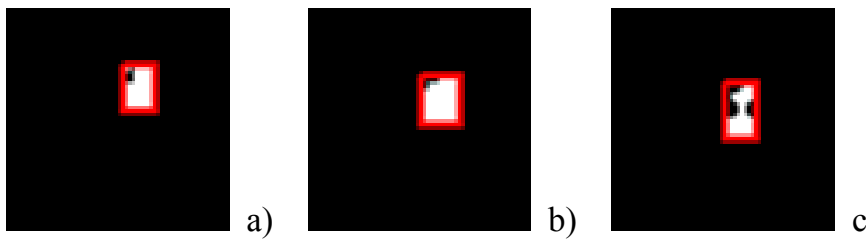

Fig. 5. Results of projection positioning.

The object trajectory can only be used for predicting a regular movement. Though this has some disadvantages, the method still meets the requirements of video surveillance. The BSCSGI tracks the moving objects in the successive two frames, where the object motion in each previous frame is taken as a prior data. The predicted fourth- and fifth-frame centroid positions are equal to $(33.0,38.5)$ and $(35.0,38.5)$, respectively. It follows from the calculations that the fourth- and fifth-frame outer rectangular boxes are 20 and 22 pixel large. The relevant simulation results are displayed in Fig. 6 . Here the range obtained by the prediction contains completely the moving object. When the sampling ratio of the moving object is $30 \%$, the outline of the object is clearly visible and the details of the target object become clear when the sampling ratio reaches $50 \%$. This means that the object tracking has been implemented successfully.

In our simulation experiments performed for the BSCSGI, the object detection efficiency increases notably whenever the object image is obtained at low-sampling ratios. We notice again 


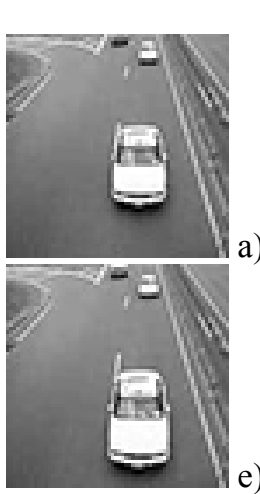

$20 \%$

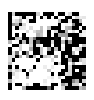

b)

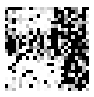

f)
$30 \%$

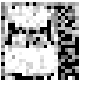

c)

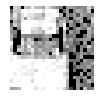

g)
$50 \%$

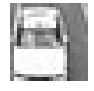

d)

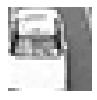

h)

Fig. 6. Results of small-scale compressive GI. Panels (a) and (e) correspond to the fourth and fifth frames, and panels (b) and (f), (c) and (g), and (d) and (h) to different sampling ratios used when reconstructing the image. The sampling ratio is equal to $20 \%$ in panels (b) and (f)), $30 \%$ in (c) and (g), and $50 \%$ in (d) and (h).

that the detected object image suffers projection positioning to obtain the object centroid. First the image is transformed into a binary image, where a standard technology of digital-image processing is used. Imperfect parts are filtered out at this stage so that the effect of noise is reduced (see Fig. 5). Then the accuracy of the projection positioning is improved, and the object trajectory found with the polynomial fit becomes more accurate. The location of the object can be predicted by the object trajectory, whereas the reconstruction methods are aimed only at the object regions. Compared with the whole-image reconstruction, the small-range image reconstruction reduces the burdens of transmission, storage and sampling ratio. Our simulation experiments performed for the object detection and object tracking have verified feasibility of the object tracking under conditions of low-sampling ratio. Summing up, the BSCSGI reduces greatly the number of samplings, alleviates the burden of transmission and storage, improves the efficiency of movingobject tracking and solves the problems of tracking under harsh conditions. These are the main advantages if compared with the common object-tracking methods.

\subsection{CSGI-based image reconstructed as compared with GI}

The GI reveals a great potential if compared with the standard imaging. In particular, this refers to the imaging of objects located in optically harsh or noisy environments. The method provides also a solution for the moving-object tracking in harsh environments. As a consequence, the GI and the CSGI are used to reconstruct the images in our simulation experiments and compare the performance of the two methods.

The experiments illustrated in Fig. 7 and Fig. 8 suggest the following.

(1) A noticeable noise is peculiar for the target image detected with the GI and, hence, the information concerned with spatial intensity distribution in the image is difficult to distinguish.

(2) The target object detected with the CSGI is clear in the case of 2000 samplings, while the target object detected with the GI is still covered by noise. Fig. 8 shows clearly that, with the same sampling, the target image detected with the CSGI includes less noise, the information distribution in the target image is more accurate, and the edges of the object are clearer. The overall result is far better than that detected using the GI method.

(3) The mean-square errors for the target images detected with both the GI and the CSGI methods show a downtrend with increasing sampling number. This demonstrates that, as the number of samplings increases, the quality of the target image detected using the target-detection methods is improved. 


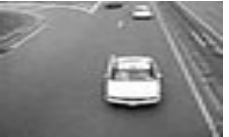

a)

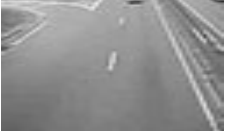

b)

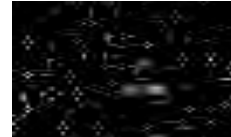

c)

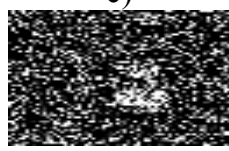

f)

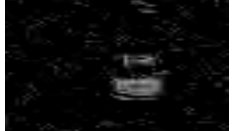

d)

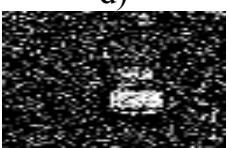

g)

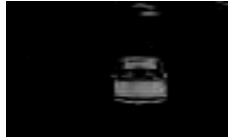

e)

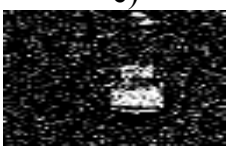

h)

Fig. 7. Illustrations of performance of the $\mathrm{GI}$ and CSGI techniques for the numbers of samplings equal to 1000 , 1500 and 2000: (a) test image, (b) background image, (c), (d) and (e) target images detected with CSGI, and (f), (g) and (h) target images detected with GI. The number of samplings is equal to 1000 in panels (c) and (f), 1500 in (d) and (g), and 2000 in (e) and (h).

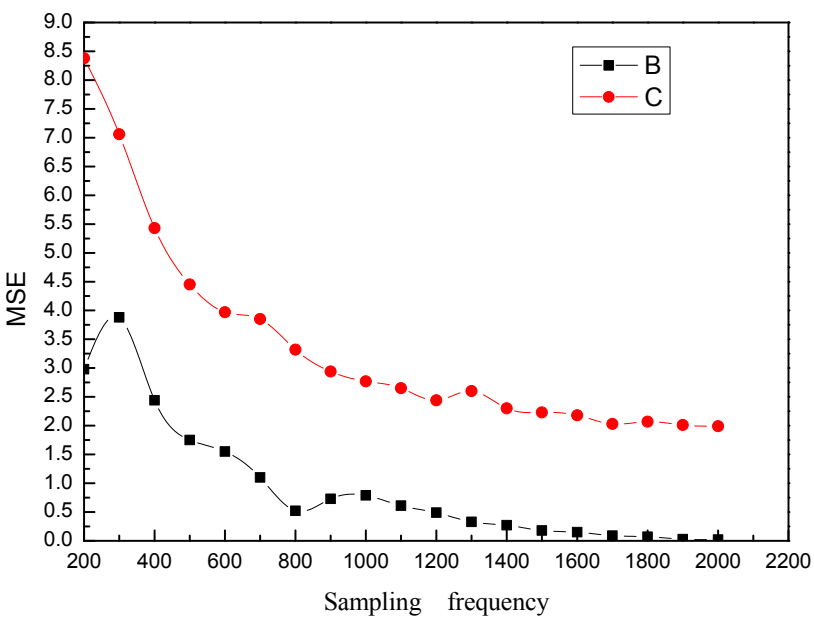

Fig. 8. Dependences of mean-square errors on the sampling frequency, as calculated for CSGI (black squares) and $\mathrm{GI}$ (red circles) techniques.

(4) The mean-square errors found for the case of CSGI are evidently less than those typical for the GI. When the sampling number reaches a certain threshold, the mean-square errors tend to become gentler. Since the mean-square errors for the target image detected with the GI are higher, the reconstructed images have also larger errors. Therefore the performance of CSGI detection is better than that of GI. Furthermore, the image quality is enhanced and the accuracy of target detection is also improved. This provides a priori knowledge for the projection positioning and the object tracking.

\subsection{BSCSGI as compared with region-based object tracking}

Among the methods of object tracking, the region-based object tracking is, maybe, the most common. Therefore we have performed simulation experiments to verify the object-tracking performance of the BSCSGI and the region-based tracking. Prior to the object tracking, the target object should be detected. Both of the methods use the BS to detect the object. The following can be concluded from the data presented in Fig. 9 and Fig. 10.

(1) Under conditions of high sampling ratios, the object obtained with the BSCSGI is clearer than that obtained with the BS. With declining sampling ratio, the BSCSGI method appears slightly noisier, although the edges of the target objects can still be accurately determined. On the other hand, the target objects detected with the BS are vague and even harder to discern the object. 


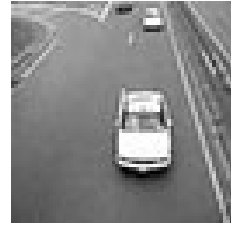

a)

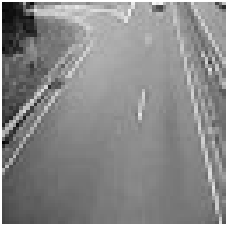

b)

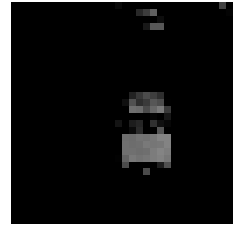

c)

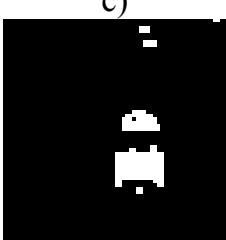

f)

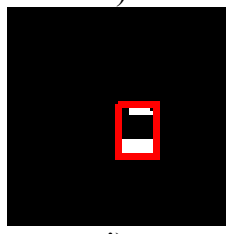

i)

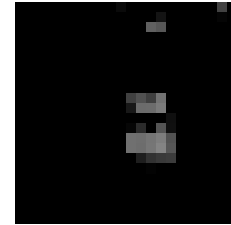

d)

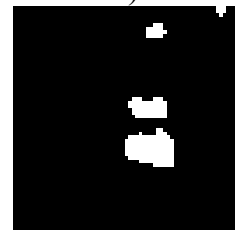

g)

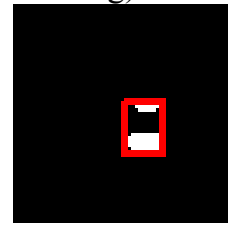

j)

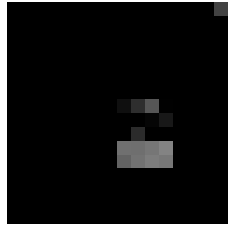

e)

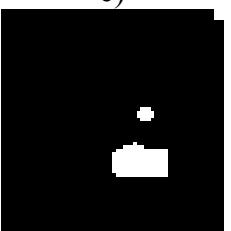

h)

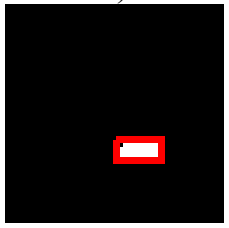

k)

Fig. 9. Simulation results obtained for the case of traditional background subtraction: (a) background image, (b) test image, (c)-(e) target object detected using the background subtraction, (f)-(h) binary images, and (i)-(k) object-region images. The sampling ratio is equal to $25 \%$ in panels (c), (f) and (i), $10 \%$ in (d), (g) and (j), and $5 \%$ in (e), (h) and (k).

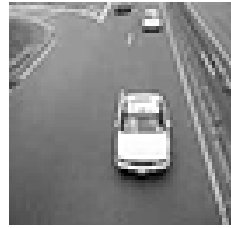

a)

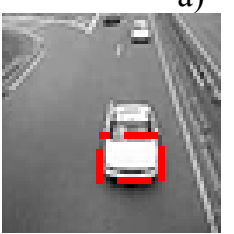

d)

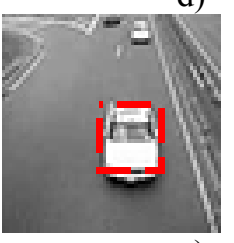

g)

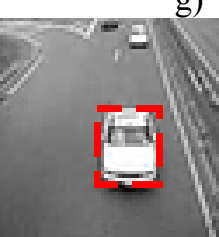

j)
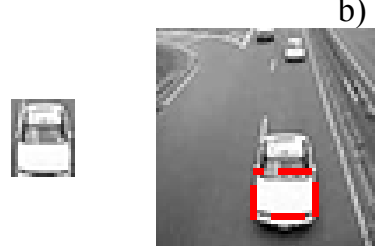

e)

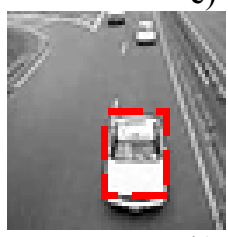

h)

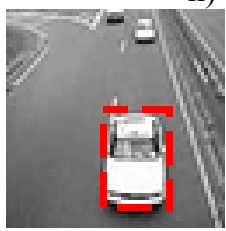

k)

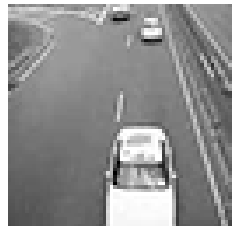

c)
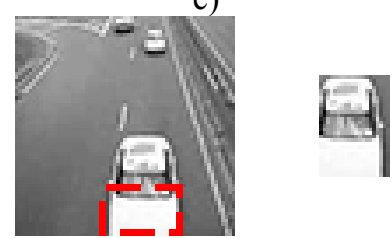

f)
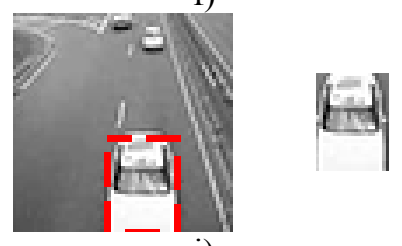

i)

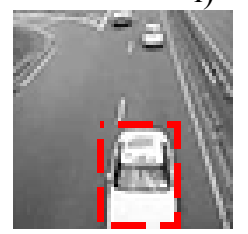

1)

Fig. 10. Comparison of tracking results obtained using region-based and BSCSGI techniques: Panels (a), (b) and (c) correspond to three consecutive-frame images, and left and right sides of panels (d)-(I) are results of the region-based object tracking and the BSCSGI, respectively. The sampling ratio is equal to $5 \%$ in panes (d), (e) and (f), $25 \%$ in $(\mathrm{g}),(\mathrm{h})$ and $(\mathrm{i})$, and $50 \%$ in $(\mathrm{j}),(\mathrm{k})$ and $(\mathrm{l})$. 
(2) When the images are binary-transformed, the technique developed in the present work can still be successfully employed to find the object at low-sampling ratios. Under the same conditions, the traditional technique is unable to identify the object accurately.

(3) With the projection positioning used to determine the object region, our technique can recognize the region where the object is located at low enough sampling ratios. On the contrary, the object region obtained using the traditional technique is deformed, and it is difficult to obtain the accurate target-area template. The latter problem can cause a loss of the target object in the tracking process, especially in the case of region-based tracking.

In case if several preceding frames serve as a prior knowledge, the objects are tracked at different sampling ratios. We have verified the tracking performance of the two techniques and the experiments illustrated in Fig. 10 and Fig. 11 prove the following facts.

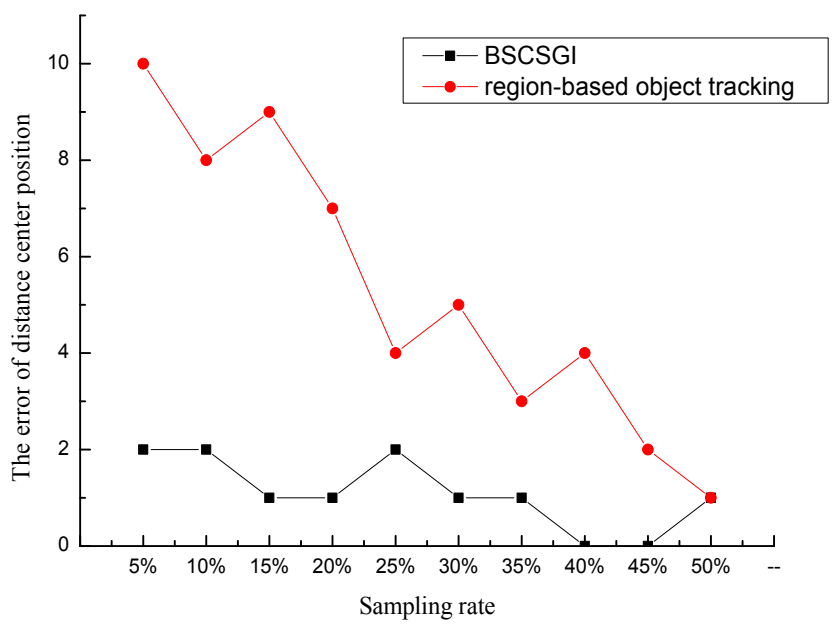

Fig. 11. Dependences of tracking errors on the sampling rate, as calculated for BSCSGI (black squares) and regionbased object-tracking (red circles) techniques.

(1) The objects detected under conditions of low-sampling ratios are used as templates for the tracking. The object-tracking results derived with the BSCSGI are better than those of the regionbased tracking. In particular, the error of our tracking technique is within acceptable range. On the other hand, the region-based technique has some difficulties when tracking accurately the targets at low-sampling ratios and larger errors are involved in this case.

(2) Both of the tracking results are evidently improved with increasing sampling ratio. The technique suggested in the present work is more stable in this respect.

(3) The tracking results derived with the BSCSGI represent small-region images so that the data redundancy is reduced and the tracking efficiency improved.

It is also seen from our simulation experiments that, in the aspect of target-object detection, the BSCSGI can detect the objects under low sampling-ratio conditions and is able to pinpoint the location of the object. In the aspect of object tracking, our technique always achieves the aim of tracking since it uses the object images detected at lower-sampling ratios to accurately predict the location of the object. Compared with the traditional object tracking, our technique reduces the sampling ratio and improves the object-tracking efficiency.

\section{Conclusion}

This study introduces the technique aimed at object tracking, which is based on combination of the compressive GI and the BS. The target image is captured using the GI and the measured characteristic of the object is obtained with the BS in the compressed domain. Then the object 
image is reconstructed directly using the CS. The centroid of the object is obtained by the projection positioning and the polynomial curve is used to fit the object trajectory. The next-frame location of the object can easily be determined and the scope of the target object is far less than the whole image. The object image is reconstructed with the compressive GI to achieve the main purpose of tracking.

The efficiency of our technique has been verified through numerical simulation experiments. We have proven that the BSCSGI can use the sampling frequencies far lower than the Nyquist sampling restrictions to restore the target objects, and so it can track the objects with high enough accuracy. The sampling number is then reduced and the tracking efficiency improved.

\section{References}

1. Kajiki J, Ochi M, Ikeya T and Shite K, 2015. Moving object tracking apparatus, and moving object tracking method. Astronomy \& Astrophys. 327: 90-106.

2. He Z, Yi S, Cheung Y M, You X and Tang Y Y, 2017. Robust object tracking via key patch sparse representation. IEEE Trans. Cybernetics. 47: 354-364.

3. Shen Y, Hu W, Yang M, Liu J, Wei B, Lucey S and Chou C T, 2016. Real-time and robust compressive background subtraction for embedded camera networks. IEEE Trans. Mobile Computing. 15: 406-418.

4. Sun T, Xing F, Wang X, Li J, Wei M and You Z, 2016. Effective star tracking method based on optical flow analysis for star trackers. Appl. Opt. 55: 10335-10340.

5. Han G, Wang J and Xi C, 2016. Background subtraction based on three-dimensional discrete wavelet transform. Sensors. 16: 456.

6. Chi-Man Pun, Caiping Yan, Xiao-Chen Yuan, 2017. Image alignment based on multi-region matching for object-level tampering detection. IEEE Transactions on Information Forensics and Security, 12: $377-391$.

7. Prabhakar N, Vaithiyanathan V, Sharma A P and Singh A, 2012. Object tracking using frame differencing and template matching. Res. J. Appl. Sci. Eng. \& Technol. 4: 5497-5501.

8. Shi H, Zhu H, Wang J, Yu S Y, Fu Z F, 2016. Segment-based adaptive window and multifeature fusion for stereo matching. J. Algorithms \& Comput. Technol. 10: 184-200.

9. Yasukawa S, Okuno H, Ishii K and Yagi T, 2016. Real-time object tracking based on scaleinvariant features employing bio-inspired hardware. Neural Networks. 81: 29-38.

10. Donoho D L, 2006. Compressed sensing. IEEE Trans. Information Theory. 52: 1289-1306.

11. Leihong Z, Bei L, Dong Land Xiuhua M, 2016. Study on the key technology of spectral reflectance reconstruction based on the weighted measurement matrix. Laser Phys. 26: 075202.

12. Zhang L, Liang D, Zhang D, Gao X and Ma X, 2016. Study of spectral reflectance reconstruction based on an algorithm for improved orthogonal matching pursuit. MRS Adv. 20: 515-523.

13. Leihong Z, Dong L, Bei L, Yi K, Zilan P, Dawei Z and Xiuhua Ma, 2016. The study of key technology on spectral reflectance reconstruction based on the algorithm of adaptive compressive sensing. Laser Phys. 26: 045201.

14. Zhu J, Ma Y, Qin Q and Zheng C, 2014. Adaptive weighted real-time compressive tracking. IET Computer Vision. 8: 740-752.

15. Yu W K, Yao X R, Liu X F, Li L Z and Zhai G J, 2015. Compressive moving target tracking with thermal light based on complementary sampling. Appl. Opt. 54: 4249-4254. 
16. Gatti A, Brambilla E, Bache M and Lugiato L A, 2004. Ghost imaging with thermal light: comparing entanglement and classical correlation. Phys. Rev. Lett. 93: 093602.

17. Magaña-Loaiza O S, Howland G A, Malik M and Howell J C, 2013. Compressive object tracking using entangled photons. Appl. Phys. Lett. 102: 231104-4.

18. Zhe Yang, Omar S. Magana-Loaiza, Mohammad Mirhosseini, Yiyu Zhou, Boshen Gao, Lu Gao, Seyed Mohammad Hashemi Rafsanjani, Guilu Long, Robert W. Boyd, 2016. Digital spiral object identification using random light. Preprint. arXiv:1609.08741.

19. Morais A P, Cardoso G, Mariotto L and Ferreira G D, 2011. Numerical distance relaying algorithm based on mathematical morphology and least-squares Ccurve fitting method. Electric Power Syst. Res. 81: 1144-1150.

Zhang Leihong, Kang Yi, Li Bei, Zhan Wenjie, Zhang Dawei and Ma Xiuhua. 2017. Studies on the key methods for compressive ghost-image tracking based on background subtraction. Ukr.J.Phys.Opt. 18: 143 - 155

Анотація. Ефективне відстеження об'єктів - ие технологія, важлива для багатьох практичних застосувань комп'ютерного зору. Відомо, що підхід «зображення 3 примарами» (ПЗ) має значний потенціал, порівняно зі стандартним відстеженням об'єктів, i вирішує багато проблем у разі, якщо традиційне відстеження об'єкта неможливе. У иій праці ми показусмо, як можна відстежувати об'єкти за методами стиснення ПЗ і вилучення фону. Спочатку фіксують інформацію про об'єкт за допомогою ПЗ. Характеристику, виміряну для об'єкта, одержують вилученням фону в стиснутій області. Ця характеристика використовує стиснене впізнавання для реконструкиії зображення об'єкта. Далі зображення об'єкта проекційно позииіонують так, щоб отримати відповідні координати центроїда. Нарешті, траєкторію об'єкта відновлюють за допомогою поліноміальної апроксимації, що забезпечує успішне відстеження об'єкта. Намі експерименти засвідчують, що ия методика здатна точно відстежсувати об'єкти за умови низьких коефіцієнтів вибірки. Крім того, вона різко зменшує кількість вимірювань, потрібних для відновлення зображення, і покращує ефективність відстеження. 